\title{
Maximizing spectral flux from self-seeding hard x-ray free electron lasers
}

\author{
Xi Yang* \\ Brookhaven National Laboratory, Upton, New York 11973, USA \\ Yuri Shvyd'ko \\ Advanced Photon Source, Argonne National Laboratory, Argonne, Illinois 60439, USA
}

(Received 20 September 2013; published 2 December 2013)

\begin{abstract}
Fully coherent $\mathrm{x}$ rays can be generated by self-seeding x-ray free electron lasers (XFELs). Self-seeding by a forward Bragg diffraction (FBD) monochromator has been recently proposed [G. Geloni, V. Kocharyan, and E. Saldin, J. Mod. Opt. 58, 1391 (2011)] and demonstrated [J. Amann et al., Nat. Photonics 6, 693 (2012)]. Characteristic time $\mathcal{T}_{0}$ of FBD determines the power, spectral, and time characteristics of the FBD seed [Yu. Shvyd'ko and R. Lindberg, Phys. Rev. ST Accel. Beams 15, 100702 (2012)]. Here we show that for a given electron bunch with duration $\sigma_{\mathrm{e}}$ the spectral flux of the self-seeding XFEL can be maximized, and the spectral bandwidth can be respectively minimized by choosing $\mathcal{T}_{0} \sim$ $\sigma_{\mathrm{e}} / \pi$ and by optimizing the electron bunch delay $\tau_{e}$. The choices of $\mathcal{T}_{0}$ and $\tau_{e}$ are not unique. In all cases, the maximum value of the spectral flux and the minimum bandwidth are primarily determined by $\sigma_{\mathrm{e}}$. Two-color seeding takes place if $\mathcal{T}_{0} \ll \sigma_{\mathrm{e}} / \pi$. The studies are performed, for a Gaussian electron bunch distribution with the parameters, close to those used in the short-bunch ( $\sigma_{\mathrm{e}} \simeq 5 \mathrm{fs}$ ) and long-bunch $\left(\sigma_{\mathrm{e}} \simeq 20 \mathrm{fs}\right)$ operation modes of the Linac Coherent Light Source XFEL.
\end{abstract}

DOI: 10.1103/PhysRevSTAB.16.120701

PACS numbers: 41.50.+h, 41.60.Cr, 61.05.cp, 42.55.Vc

\section{INTRODUCTION}

Seeded x-ray free electron lasers (XFELs) [1,2] generate fully coherent $x$ rays with a well-defined spectrum and higher spectral flux. Their supreme spectral properties will expand the science reach of the XFELs, and stimulate the utilization of advanced high-resolution spectroscopic techniques. The self-seeding XFEL is also a path to increase the peak radiation power of XFELs to the TW levels [3-11]. In the present paper we study the conditions for stable seeding, with the smallest shot-to-shot variations of output radiation, the narrowest bandwidth, and largest spectral flux.

The self-seeding scheme uses $x$ rays from the first half of the FEL system (electron beam-magnetic undulator system-radiation field) to generate radiation by the selfamplified spontaneous emission (SASE) process [12-16] to seed the electron bunch in the second half of the FEL system via an $\mathrm{x}$-ray monochromator $[17,18]$. The delay of $\mathrm{x}$ rays in the monochromator is compensated by the appropriate delay $\tau_{e}$ of the electron bunch in the magnetic chicane. Standard $\mathrm{x}$-ray monochromators produce delays in the picosecond range, at least. In the hard $\mathrm{x}$-ray regime with high-energy (10-20 GeV) electrons, a large magnetic

*xiyang@bnl.gov

†shvydko@aps.anl.gov

Published by the American Physical Society under the terms of the Creative Commons Attribution 3.0 License. Further distribution of this work must maintain attribution to the author(s) and the published article's title, journal citation, and DOI. chicane is required to match the path-length delay of the $\mathrm{x}$-ray monochromator, which may result in generation of incoherent and coherent synchrotron radiation that may degrade the electron-beam qualities. A possible solution is using a two-electron-bunch self-seeding scheme [19,20]. Another elegant solution, proposed by Geloni, Kocharyan, and Saldin, uses forward Bragg diffraction (FBD) of x rays from a diamond crystal to generate a monochromatic seed [21]-Fig. 1. The first trailing maximum of FBD was proposed for the self-seeding of $\simeq 5$-fs $(\mathrm{FWHM})$ short electron bunches, at an optimal $\approx 20$-fs delay of the seed relative to the electron bunch. The proposal has been recently realized and fully coherent hard $\mathrm{x}$ rays have been generated at the Coherent Light Source (LCLS) XFEL by an international team led by Emma [1].

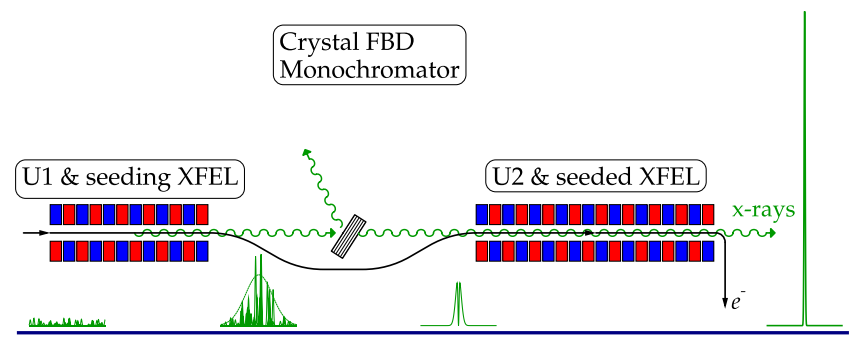

FIG. 1. Hard x-ray FEL self-seeding scheme uses $x$ rays from the first half of the magnetic undulator system U1 to seed the electron bunch in the second half $\mathrm{U} 2$ via a single crystal $\mathrm{x}$-ray monochromator. The monochromator produces the delayed monochromatic seed under the forward Bragg diffraction (FBD) conditions [21]. 

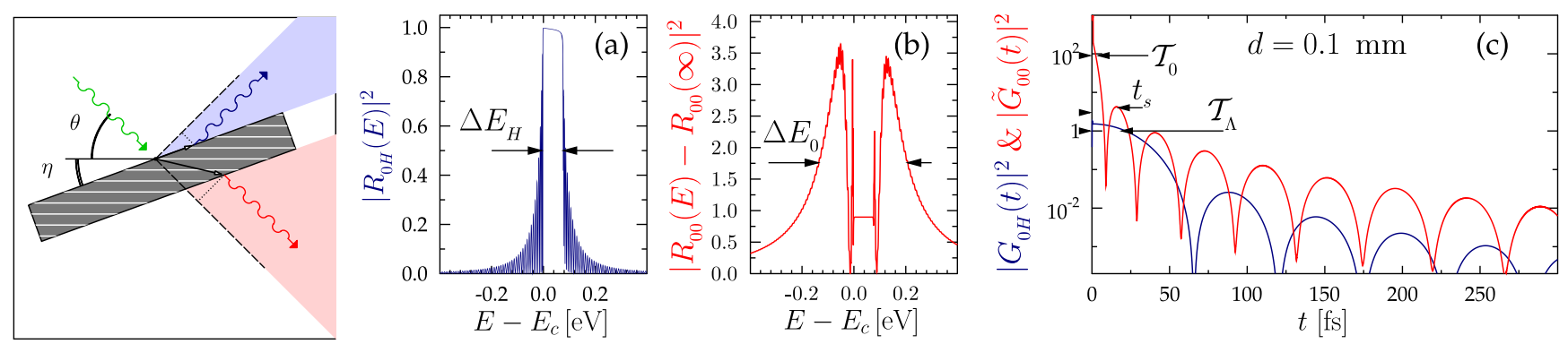

FIG. 2. (left) Schematic of Bragg diffraction (BD) and forward Bragg diffraction (FBD) of x rays from a crystal in Bragg-case geometry. (a) Example of energy dependence of x-ray reflectivity $\left|R_{0 H}(E)\right|^{2}$ in Bragg diffraction and (b) of energy dependence of forward Bragg diffraction $\left|R_{00}(E)-R_{00}(\infty)\right|^{2}$ from a diamond crystal in the 004 Bragg reflection, $E_{\mathrm{c}}=8.33 \mathrm{keV}, \theta=56.6^{\circ}$, $\eta=0^{\circ}, d=0.1 \mathrm{~mm}$. (c) Intensities of corresponding time dependencies of crystal response to an excitation with ultrashort x-ray pulse in $\mathrm{BD}\left|G_{0 H}(t)\right|^{2}$ (blue) and in $\operatorname{FBD}\left|G_{00}(t)\right|^{2}$ (red), respectively.

The question that naturally arises is: Under what conditions is seeding most efficient in terms of achieving the shortest XFEL saturation length, the smallest spectral bandwidth of output radiation, and the largest spectral density? One could expect that for this, generally speaking, the monochromatic seed duration should be comparable to the duration of the electron pulse, the monochromatic seed power should substantially exceed the shot-noise power, and the peak seed power should overlap with the peak electron current density. The question then concretizes to which crystal, which Bragg reflection, which scattering geometry, which crystal thickness, etc. suit best? This is a problem with many parameters, which is difficult to tackle. However, there is a possibility to reduce the problem to one single parameter.

It has been shown in [22] that under a certain approximation the time dependence of FBD can be characterized by a single parameter $\mathcal{T}_{0}$, the time constant of forward Bragg diffraction. It is the main parameter that defines the strength, and duration of the FBD monochromatic seed. It is a generic feature for all symmetric or asymmetric, for all transmission (Laue case) or reflection (Bragg case) scattering geometries. The physics is controlled by the parameters that compose $\mathcal{T}_{0}$ : the magnitude of the effective crystal thickness (crystal thickness seen by the incident beam), and the extinction length in the symmetric Bragg reflection (characteristic length of Bragg diffraction). This approximation is correct if we are interested in the FBD radiation emanating from the crystal with delays $t$, which are significantly shorter than a mere propagation time $\mathcal{T}_{d}$ of the radiation through the crystal. It turns out that exactly for self-seeding applications the approximation $t \ll \mathcal{T}_{d}$ holds well in many cases.

Because of this property, the spectral flux optimization could be studied by analyzing self-seeding as a function of the principle time constant $\mathcal{T}_{0}$. With one more time constant $\mathcal{T}_{d}$ taken into account, a more accurate picture can be achieved. Here we perform such studies using the 3D timedependent FEL simulation code GENESIS 1.3 by Sven Reiche [23].
The paper is organized as follows. In Sec. II we briefly summarize the basic properties of Bragg diffraction (BD) and the complementary to it forward Bragg diffraction (FBD), and outline the procedure to calculate the monochromatic seed in forward Bragg diffraction from a crystal in the XFEL self-seeding scheme. In Sec. III we provide details on the 3D FEL simulations. In Sec. IV we present and discuss the results of the simulations.

\section{GENERATION OF THE MONOCHROMATIC SEED IN FBD}

\section{A. Basic properties of forward Bragg diffraction}

Here we briefly summarize the basic properties of Bragg diffraction (FB) and of the complementary forward Bragg diffraction (FBD); define their characteristic spectral, time, and space parameters; and outline, using results and notations of [22], the procedure for calculating the monochromatic seed in FBD from a crystal in the XFEL self-seeding scheme.

Bragg diffraction (BD) of $\mathrm{x}$ rays incident upon a crystal at a glancing angle $\theta$ to reflecting atomic planes with diffraction vector $\boldsymbol{H}$, see Fig. 2 (left), takes place within a spectral range $\Delta E_{H}$ centered at a photon energy $E_{\mathrm{c}}=$ $H c \hbar / 2 \sin \theta$, defined by Bragg's law. Here $c$ is the speed of light in vacuum, and $\hbar$ is Planck's constant. An example of Bragg reflection spectral dependence $\left|R_{0 H}(E)\right|^{2}$ is shown in Fig. 2(a). All photons within the Bragg reflection spectral range $\Delta E_{H}$ are almost totally reflected from the crystal and propagate at a scattering angle $2 \theta$ with a characteristic delay time $\mathcal{T}_{\Lambda}=2 \hbar / \Delta E_{H}$ of Bragg diffraction, as shown in Fig. 2(c). In fact, $\mathcal{T}_{\Lambda}$ scales with the characteristic length of Bragg diffraction $\bar{\Lambda}_{H}$, the so-called extinction length:

$$
\mathcal{T}_{\Lambda}=\frac{2 \bar{\Lambda}_{H} \sin ^{2} \theta}{c\left|\gamma_{H}\right|}, \quad \mathcal{T}_{\Lambda} \Delta E_{H}=2 \hbar
$$

Here $\gamma_{H}=\sin (\eta-\theta)$, and $\eta$ is an asymmetry angle, see Fig. 2 (left). 

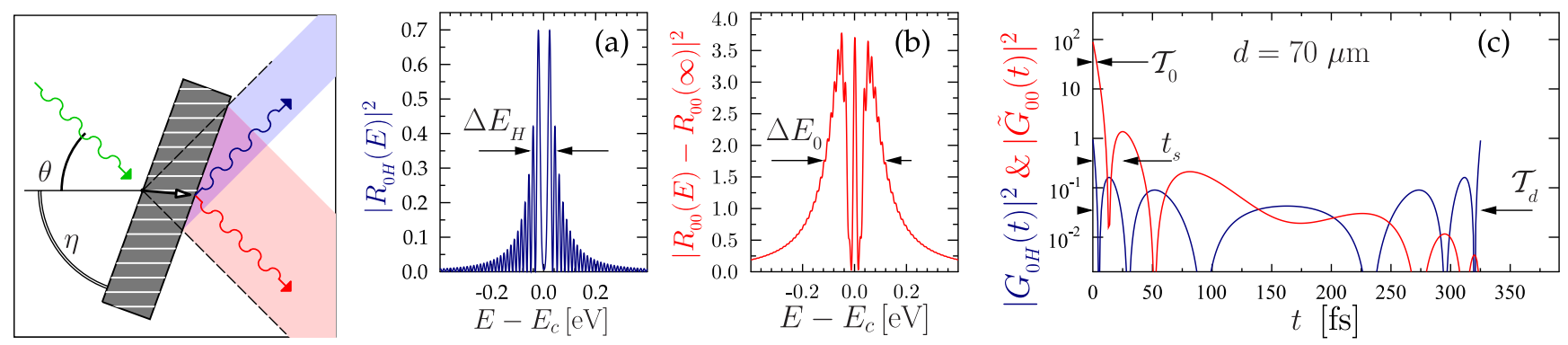

FIG. 3. (left) Schematic of Bragg diffraction (BD) and forward Bragg diffraction (FBD) of $x$ rays from a crystal in Laue-case scattering geometry. All notations are as in Fig. 2. Example of energy and time dependencies from a diamond crystal in the 004 Bragg reflection, $E_{\mathrm{c}}=8.33 \mathrm{keV}, \theta=56.6^{\circ}, \eta=90^{\circ} d=0.1 \mathrm{~mm}$.

Forward Bragg diffraction (FBD) is a multiple coherent scattering of $\mathrm{x}$ rays from the same atomic planes. It is complementary to the BD scattering process. Those spectral components of the incident radiation contribute to FBD, which do not participate in BD, those which are outside but in the immediate vicinity of the Bragg reflection region $\Delta E_{H}$. The FBD spectral dependence $\mid R_{00}(E)-$ $\left.R_{00}(\infty)\right|^{2}$ in Fig. 2(b) illustrates this. Here $R_{00}(E)$ is a transmission amplitude through the crystal of a spectral component with energy $E$. The FBD spectral width $\Delta E_{0}$ scales with the BD spectral width $\Delta E_{H}$, is larger than $\Delta E_{H}$, and increases with the crystal thickness $d$ :

$\Delta E_{0}=\Delta E_{H} \frac{d}{2 \pi \bar{\Lambda}_{H}}, \quad \mathcal{T}_{0}=\mathcal{T}_{\Lambda} \frac{\bar{\Lambda}_{H}}{d}, \quad \mathcal{T}_{0} \Delta E_{0}=\frac{\hbar}{\pi}$.

Respectively, the characteristic time of FBD $\mathcal{T}_{0}$ see Fig. 2(c), scales with $\mathcal{T}_{\Lambda}$, is smaller than $\mathcal{T}_{\Lambda}$, and decreases with the crystal thickness.

The FBD crystal response to the excitation by an incident radiation pulse can be calculated as a convolution in time of the incident SASE radiation pulse amplitude $\mathcal{E}_{\text {SASE }}(t)$ and of a forward Bragg diffraction response function $G_{00}$ :

$$
\mathcal{E}_{0}(t)=\int_{-\infty}^{t} \mathrm{~d} t^{\prime} G_{00}\left(t-t^{\prime}\right) \mathcal{E}_{\mathrm{SASE}}\left(t^{\prime}\right) .
$$

Here $\mathcal{E}_{\mathrm{SASE}}(t)$ is a slowly varying envelope. $G_{00}(t)$ presents the crystal response to a delta-function excitation in time and is calculated as a Fourier transform of the FBD spectral amplitudes:

$$
\begin{aligned}
& G_{00}(t)=R_{00}(\infty)\left[\delta(t)+\tilde{G}_{00}(t)\right] \\
& \tilde{G}_{00}(t)=\int_{-\infty}^{\infty} \frac{\mathrm{d} \Omega}{2 \pi} \mathrm{e}^{-\mathrm{i} \Omega t} \frac{R_{00}(\Omega)-R_{00}(\infty)}{R_{00}(\infty)},
\end{aligned}
$$

where $\Omega=\left(E-E_{\mathrm{c}}\right) / \hbar$. According to Eq. (4), $G_{00}(t)$ is in fact a sum of the prompt, i.e., diffraction-free transmission $R_{00}(\infty) \delta(t)$ of the far-from-Bragg-reflection-region spectral components, and of the delayed actual forward diffraction response function $\tilde{G}_{00}(t)$. As a result, the final expression for the time dependence of the radiation field emanating from the crystal in the forward direction,

$\mathcal{E}_{0}(t)=R_{00}(\infty)\left[\mathcal{E}_{\mathrm{SASE}}(t)+\int_{-\infty}^{t} \mathrm{~d} t^{\prime} \tilde{G}_{00}\left(t-t^{\prime}\right) \mathcal{E}_{\mathrm{SASE}}\left(t^{\prime}\right)\right]$

is a sum of the diffraction-free transmitted SASE radiation and of the second component, which is the delayed monochromatic seed due to FBD.

In general, FBD is characterized also by a spatial transverse shift of the delayed radiation emitted by the crystal in the forward direction [22,24]. In the present studies we will, however, assume for simplicity that the spatial shift of the FBD signal is small and can be neglected.

An analytical expression can be derived for the forward diffraction time response function $\tilde{G}_{00}(t)$ in the approximation of a nonabsorbing and thick $d \gg \bar{\Lambda}_{H}^{(\mathrm{s})}$ crystal valid in the general case of asymmetric Bragg diffraction, both in Bragg-case geometry (Fig. 2):

$$
\tilde{G}_{00}(t)=-\frac{1}{2 \mathcal{T}_{0}} \frac{J_{1}\left[\sqrt{\frac{t}{\mathcal{T}_{0}}\left(1+\frac{t}{\mathcal{T}_{d}}\right)}\right]}{\sqrt{\frac{t}{\mathcal{T}_{0}}\left(1+\frac{t}{\mathcal{T}_{d}}\right)}},
$$

and in Laue-case geometry (Fig. 3):

$$
\begin{gathered}
\tilde{G}_{00}(t)=\frac{1}{2 \mathcal{T}_{0}}\left(1-\frac{t}{\mathcal{T}_{d}}\right) \frac{J_{1}\left[\sqrt{\frac{t}{\mathcal{T}_{0}}\left(1-\frac{t}{\mathcal{T}_{d}}\right)}\right]}{\sqrt{\frac{t}{\mathcal{T}_{0}}\left(1-\frac{t}{\mathcal{T}_{d}}\right)}} \\
{\left[0<t<\mathcal{T}_{d}\right],}
\end{gathered}
$$

with the characteristic time parameters

$$
\mathcal{T}_{0}=\frac{2\left[\bar{\Lambda}_{H}^{(\mathrm{s})}\right]^{2}}{c\left(d / \gamma_{0}\right)}, \quad \mathcal{T}_{d}=\frac{2 d \sin ^{2} \theta}{c\left|\gamma_{H}\right|} .
$$

$\mathcal{T}_{0}$ is the characteristic time of forward Bragg diffraction, the same as in Eq. (2). Here it is expressed, however, through a Bragg-reflection invariant - the extinction length $\bar{\Lambda}_{H}^{(\mathrm{s})}$ in symmetric diffraction geometry $(\eta=0)$-and by the effective crystal thickness $d / \gamma_{0}$, the thickness seen by 


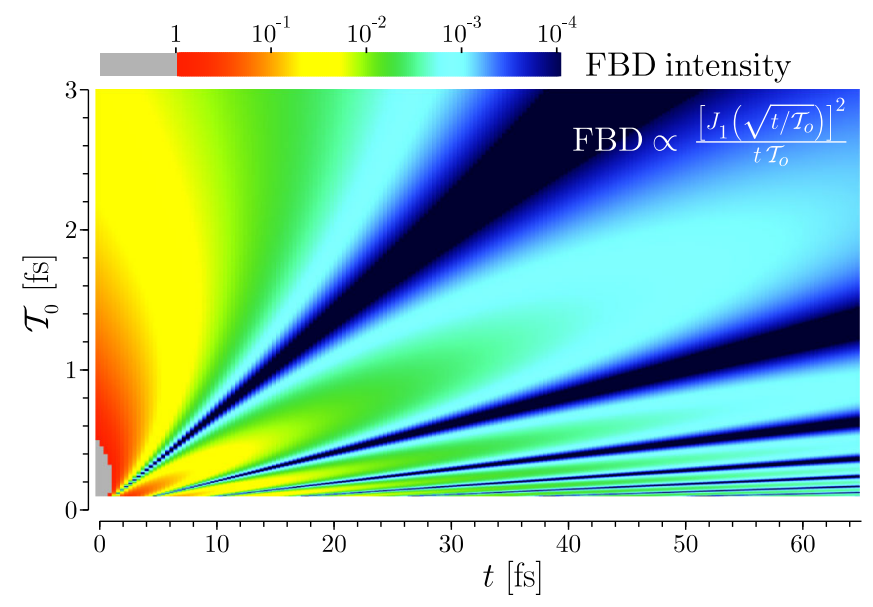

FIG. 4. 2D plot of the universal FBD response function intensity $\left|\tilde{G}_{00}(t)\right|^{2}$ (in relative units) plotted for different values of the time delay $t$ to the instantaneous excitation of the crystal, and for different values of the FBD time constant $\mathcal{T}_{0}$, using Eq. (9).

incident $\mathrm{x}$ rays, $\gamma_{0}=\sin (\theta+\eta)$. Extinction length $\bar{\Lambda}_{H}^{(\mathrm{s})}$ values for Bragg reflections in diamond, $\mathrm{Si}$, and $\mathrm{Al}_{2} \mathrm{O}_{3}$ are tabulated in $[22,25]$. The parameter $\mathcal{T}_{d}$ is a characteristic measure of time in Bragg diffraction associated with the crystal thickness $d$. In the Laue-case geometry, $\mathcal{T}_{d}$ is equal to the total duration of forward Bragg diffraction, see Fig. 3(c). In the Bragg-case geometry, it measures the time between multiple reflections.

If additionally, the delay times of interest are short $t \ll \mathcal{T}_{d}$ compared to the propagation time through the

TABLE I. Examples of the time constants $\mathcal{T}_{0}$, and $\mathcal{T}_{d}$ in Eq. (8) used in the subsequent simulations; and their realization through particular (not at all unique) Bragg diffraction cases. The appropriate FBD spectral bandwidths $\Delta E_{0}-$ Eq. (2) -are also provided for reference. Each Bragg diffraction case is defined first of all by the Bragg-reflection-invariant diffraction vector $\boldsymbol{H}$ and symmetric extinction length $\bar{\Lambda}_{H}^{(\mathrm{s})}$ in diamond crystals, by the crystal thickness $d$, and by the parameters defining scattering geometry: the Bragg angle $\theta$, the asymmetry angle $\eta$, the incidence wave vector projection $\gamma_{0}=\sin (\theta+\eta)$ to the crystal internal normal, and the reflection wave vector projection $\gamma_{H}=\sin (\eta-\theta)$, respectively.

\begin{tabular}{lcrcccccccc}
\hline \hline $\boldsymbol{H}$ & $\bar{\Lambda}_{H}^{(\mathrm{s})}$ & \multicolumn{1}{c}{$d$} & $\theta$ & $\eta$ & & & & & $\Delta E_{0}$ \\
$h k l$ & $\mu \mathrm{m}$ & $\mu \mathrm{m}$ & $\operatorname{deg}$ & $\operatorname{deg}$ & $\gamma_{H}$ & \multicolumn{1}{c}{$\gamma_{0}$} & $\mathcal{T}_{0}$ fs & $\mathcal{T}_{d}$ fs & $\mathrm{meV}$ \\
\hline 111 & 1.09 & 40 & 21.3 & 0 & 0.36 & -0.36 & 0.07 & 94 & 2993 \\
111 & 1.09 & 100 & 21.3 & 90 & 0.93 & 0.93 & 0.07 & 94 & \\
220 & 1.98 & 75 & 36.3 & 0 & 0.59 & -0.59 & 0.21 & 296 & 998 \\
220 & 1.98 & 100 & 36.3 & 90 & 0.81 & 0.81 & 0.21 & 296 & \\
400 & 3.63 & 100 & 56.8 & 0 & 0.84 & -0.84 & 0.73 & 558 & 287 \\
511 & 7.83 & 260 & 82.9 & 15.8 & 0.99 & -0.92 & 1.5 & 1720 & 140 \\
511 & 7.83 & 130 & 82.9 & 15.8 & 0.99 & -0.92 & 3.0 & 860 & 70 \\
511 & 7.83 & 100 & 82.9 & 15.8 & 0.99 & -0.92 & 4.1 & 712 & 51 \\
511 & 7.83 & 58 & 82.9 & 15.8 & 0.99 & -0.92 & 7.0 & 413 & 30 \\
\hline \hline
\end{tabular}

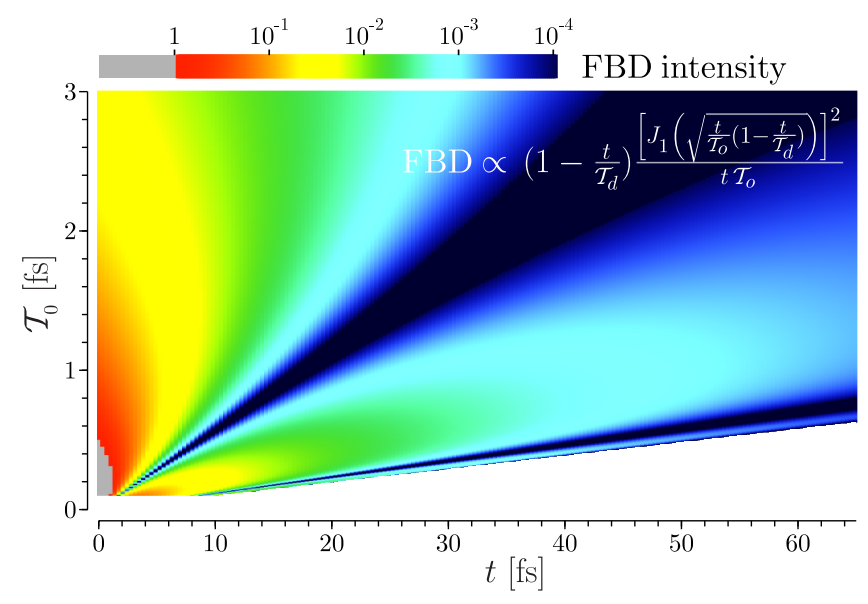

FIG. 5. Similar to Fig. 4, however, Eq. (4) for the Laue-case FBD response function is used for a particular case of $\mathcal{T}_{d} \simeq$ $104 \mathcal{T}_{0}$, which permits the duration of the FBD peaks to be stretched.

crystal, a generic expression can be used for all symmetric or asymmetric, Brag-case or Laue-case geometries:

$$
\tilde{G}_{00}(t)=\operatorname{sgn}\{b\} \frac{1}{2 \mathcal{T}_{0}} \frac{J_{1}\left(\sqrt{t / \mathcal{T}_{0}}\right)}{\sqrt{t / \mathcal{T}_{0}}}, \quad t \ll \mathcal{T}_{d}
$$

The time constant of forward Bragg diffraction $\mathcal{T}_{0}$ [Eq. (8)] is the only parameter that enters Eq. (9), and is the only parameter that defines the strength, delay, and duration of FBD and therefore of the monochromatic seed. The physics is controlled by the parameters that compose $\mathcal{T}_{0}$ [Eq. (8)]: the magnitude of the effective crystal thickness $d / \gamma_{0}$, and the extinction length $\bar{\Lambda}_{H}^{(\mathrm{s})}$ in the symmetric Bragg reflection. A $2 \mathrm{D}$ plot of the FBD intensity $\propto\left|\tilde{G}_{00}(t)\right|^{2}$, in the $\left(t, \mathcal{T}_{0}\right)$ space, shown in Fig. 4, can be used as an initial guide to the most beneficial $\mathcal{T}_{0}$ values for seeding at a given time delay $t$.

The first trailing maximum of $\left|\tilde{G}_{00}(t)\right|^{2}$, which was originally proposed to use as the monochromatic seed, appears at the time delay $t_{s}=26 \mathcal{T}_{0}$, see Fig. 2(c). Its duration is $\Delta t_{s}=16.5 \mathcal{T}_{0}$. Both the delay and duration can be tailored by changing $\mathcal{T}_{0}$, which can be done practically by adjusting the extinction length $\bar{\Lambda}_{H}^{\text {(s) }}$ (for example, by choosing another reflection or asymmetry parameter), or by changing the crystal thickness.

Table I shows examples of $\mathcal{T}_{0}$ and $\mathcal{T}_{d}$ used in the subsequent simulations and how these values can be realized with particular Bragg reflections. The realization of a particular $\mathcal{T}_{0}$ is not unique. The same $\mathcal{T}_{0}$ values can be realized with different Bragg diffraction vectors, i.e., different $\bar{\Lambda}_{H}^{(\mathrm{s})}$, different crystal thicknesses $d$, and different scattering geometry parameters $\theta, \eta, \gamma_{H}$, and $\gamma_{0}$.

An interesting situation may occur in Laue-case diffraction. Unlike the Brag-case diffraction presented by Eq. (6), 

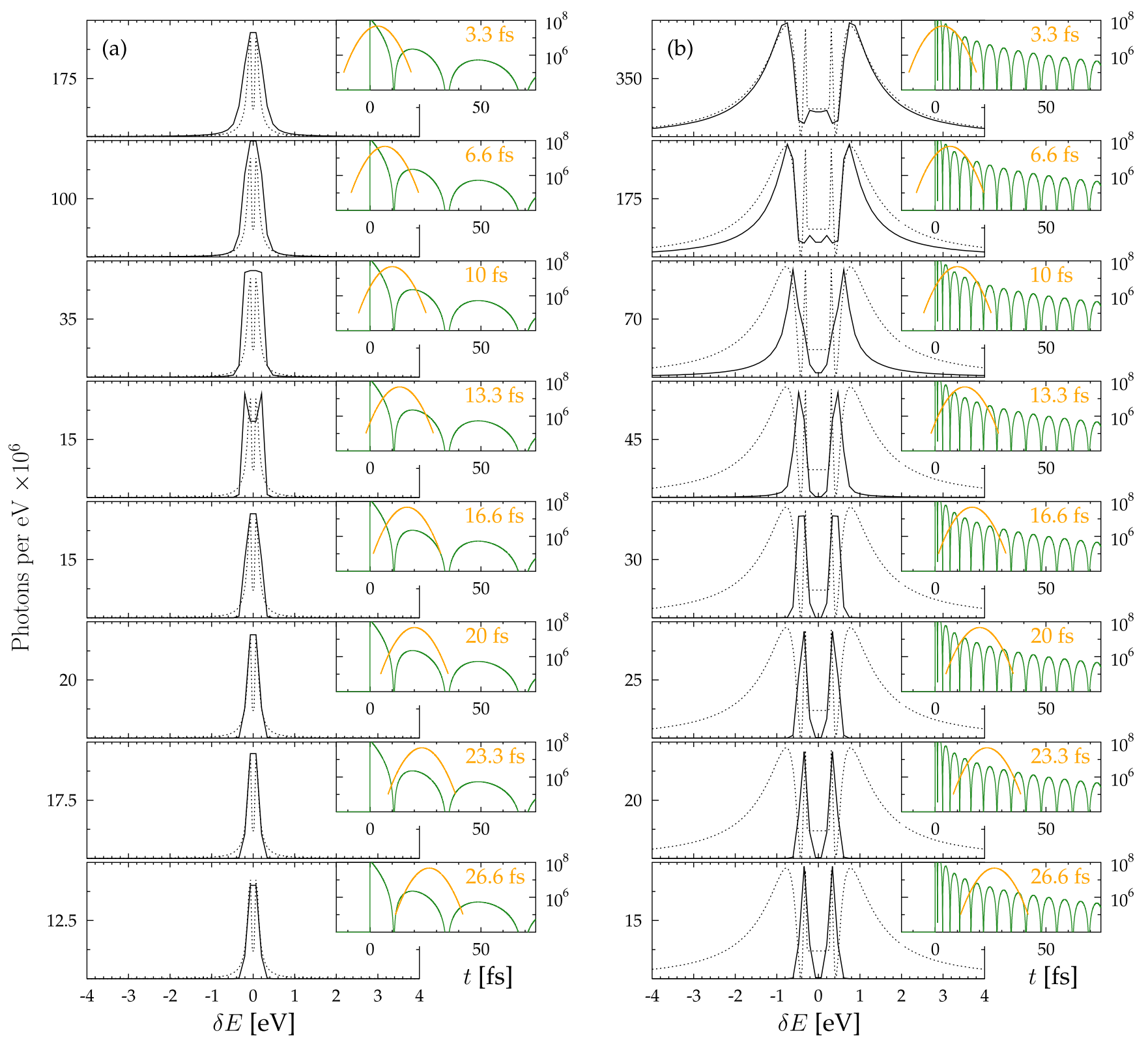

FIG. 6. Spectra of the seeding radiation as "seen" by the electron bunch (solid black lines). The spectra are calculated for different delays $\tau_{e}$ of the electron bunch (solid orange lines in the insets) with respect to the FBD intensity (solid green lines in the insets). The dotted lines show for reference the time-integrated FBD spectra. Calculations are presented for an electron bunch with an rms duration $\sigma_{\mathrm{e}}=5.3 \mathrm{fs}$, Bragg-case FBD time constant $\mathcal{T}_{0}=0.75 \mathrm{fs}$ (a), and $\mathcal{T}_{0}=0.07 \mathrm{fs}$ (b), respectively. Case (a) with $\mathcal{T}_{0} \simeq \sigma_{\mathrm{e}} / \pi$ is optimal for the single-color seeding, while case (b) with $\mathcal{T}_{0} \ll \sigma_{\mathrm{e}} / \pi$ is better suited for the two-color seeding.

the argument $a=t / \mathcal{T}_{0}\left(1-t / \mathcal{T}_{d}\right)$ of the response function in the Laue-case diffraction, Eq. (7), attains the maximum value $a_{\max }=\mathcal{T}_{d} / 4 \mathcal{T}_{0}$ at $t_{\max }=\mathcal{T}_{d} / 2$ The argument changes slowly with time around $t_{\max }$. If in addition $a_{\max } \simeq 26$, i.e., $\quad \mathcal{T}_{d} / \mathcal{T}_{0}=$ $\left(d / \bar{\Lambda}_{H}^{(\mathrm{s})}\right)^{2} \sin ^{2} \theta /\left(\left|\gamma_{H}\right| \gamma_{0}\right) \simeq 104 \mathcal{T}_{0}$, the first trailing maximum of the response function will be stretched in time compared to the Bragg-case FBD, as graphically shown in Fig. 5. This property may be useful for seeding long electron bunches.

\section{B. Single-color and multicolor seeding conditions}

The FBD spectra presented in Figs. 2(b) and 3(b) are time integrated. The actual spectrum of the radiation that seeds the electron bunch is related to it, but not identical, as the electron bunch "sees" only a part of the FBD signal. The seeding radiation spectrum is determined by the Fourier transform of the product of the FBD amplitude $\mathcal{E}_{0}(t)$-Eq. (5), and of the electron current time profile. Examples of seeding radiation spectra, calculated for an idealistic case of prompt excitation $\mathcal{E}_{\mathrm{SASE}}(t) \propto \delta(t)$ and for an electron bunch with Gaussian profile, are shown in 
Fig. 6. The radiation spectrum that seeds the electron bunch depends largely on the relationship between the electron bunch duration $\sigma_{\mathrm{e}}$ and the time profile of the radiation overlapping with the electron bunch. The latter can be varied either by the FBD time constant $\mathcal{T}_{0}$ or/and by the electron bunch delay $\tau_{e}$.

In the limiting case of a flat time profile, the seeding spectrum is defined by $\sigma_{\mathrm{e}}$ and will have a spectral width of $\simeq \hbar / \sigma_{\mathrm{e}}$. This spectral width represents the finest spectral feature that the XFEL system can generate. This is also a typical spectral width of the spikes in the SASE spectra $[16,26]$.

Therefore, if the FBD spectral width is chosen to be $\Delta E_{0} \simeq \hbar / \sigma_{\mathrm{e}}$, i.e., $\sigma_{\mathrm{e}} \simeq \pi \mathcal{T}_{0}$, as in the case presented in Fig. 6(a), a close to optimal condition for single-color seeding can be achieved.

In the other limiting case, $\Delta E_{0} \gg \hbar / \sigma_{\mathrm{e}}$, i.e., $\sigma_{\mathrm{e}} \gg$ $\pi \mathcal{T}_{0}$, the FBD time profile has a few oscillations superimposed on the electron bunch. As a result, the seeding spectrum will have a double-peak structure with the separation between the peaks inversely proportional to the oscillation period, i.e., to $\propto 1 / \mathcal{T}_{0}$. This case is presented in Fig. 6(b). The FBD spectrum seen by the electron bunch changes dramatically with time. At small time delays the two outer broader peaks of the FBD spectrum dominate. At larger time delays, the two narrow spectral peaks survive and represent the seeding spectrum. In any case, at any time, the double-peak structure persists, a prerequisite for the two-color seeding. For this, however, appropriate spectral components should also be present in the SASE spectrum. Because of the stochastic nature of the SASE spectrum, this likely will not always be the case. The XFEL output will result in a two-color output, however, with the spectral content varying from shot to shot.

The Laue-case FBD spectrum can be more fine structured, see Fig. 3(b). This may provide conditions for more than two-color seeding, i.e., multicolor seeding. Even more important, in the Laue-case geometry the fine structure may have a single narrow peak in the center (unlike the Bragg case, which always has a double-hump structure); this could be beneficial for a true single-color seeding, as the broad-band spectral components decay fast, leaving only the sharp spectral component for single-color seeding.

\section{3D FEL SIMULATION DETAILS}

The 3D time-dependent FEL simulation code GENESIS 1.3 [23] is applied to study XFEL self-seeding with the FBD (wake) monochromator. The main FEL parameters used in the simulations are presented in Table II. They are based on the LCLS commissioning results [27]. Two magnetic undulator systems are used, both with the nominal undulator period $\lambda_{w}=30 \mathrm{~mm}$. The length of the first undulator system $\mathrm{U} 1$ is chosen to ensure FEL operations in the exponential growth regime and to achieve the $\mathrm{x}$-ray seed power after the monochromator about 2 orders of magnitude larger than
TABLE II. Main XFEL parameters in self-seeding mode with two magnetic undulator systems U1 and U2, and with a FBD monochromator.

\begin{tabular}{lccc}
\hline \hline Parameter and symbol & $\begin{array}{c}\text { Long-bunch } \\
\text { mode }\end{array}$ & $\begin{array}{c}\text { Short-bunch } \\
\text { mode }\end{array}$ & Unit \\
\hline Electron energy $E_{\mathrm{e}}$ & 14.3 & 13.6 & $\mathrm{GeV}$ \\
Bunch charge & 150 & 40 & $\mathrm{pC}$ \\
Peak current $I_{\mathrm{e}}$ & 3 & 3 & $\mathrm{kA}$ \\
Slice energy spread & 2.8 & 5.6 & $\mathrm{MeV}$ \\
Slice emittance & 0.4 & 0.4 & $\mu \mathrm{m}$ \\
Bunch duration $\sigma_{\mathrm{e}}$ & $20(\mathrm{rms})$ & $5.3(\mathrm{rms})$ & $\mathrm{fs}$ \\
Bunch duration $\Delta t_{\mathrm{e}}$ & $47(\mathrm{FWHM})$ & $12.5(\mathrm{FWHM})$ & $\mathrm{fs}$ \\
U1 length & 60 & 60 & $\mathrm{~m}$ \\
U2 length & 70 & 70 & $\mathrm{~m}$ \\
Undulator period $\lambda_{w}$ & 30 & 30 & $\mathrm{~mm}$ \\
Normalized undulator & 2.4729 & 2.4729 & \\
$\quad$ parameter $a_{w}$ & & & \\
\hline \hline
\end{tabular}

the shot-noise power $P_{\mathrm{n}}$. At the same time, the U1 length is limited to $60 \mathrm{~m}$ to avoid energy spread growth due to FEL saturation. The second undulator system U2 is chosen to be $70 \mathrm{~m}$ long for the FEL to reach saturation with reduced fluctuations of the x-ray pulse power. The lengths of the first and the second magnetic undulator systems include the gaps between the undulator sections. A weak four-dipole chicane with $R_{56} \simeq 3-45 \mu \mathrm{m}$ is installed in a 4-m-long space between the $\mathrm{U} 1$ and $\mathrm{U} 2$ undulator systems.

There are typical shot-to-shot SASE radiation fluctuations after the U1 undulator system. As a result, the power and phase of the radiation after the monochromator fluctuate as well, as shown in Fig. 7. See also the discussion in [24]. The power fluctuations could be about $50 \%$. There is a $\simeq 10-15$-fs jitter in the duration and in the form of the SASE signal, resulting in a similar jitter of the seed oscillation peak positions and of the seed phase. The phase time dependences appear to be very different from shot to shot, which is related to how the convolution of a particular SASE radiation pulse with the crystal response function works, see Eq. (5). For realistic FEL output, averaging is required over many shorts. The averaged picture, however, may obscure the underlying physics. For this reason, the following analysis is performed without averaging, but using instead a single typical SASE radiation pulse for all cases. In particular, the SASE pulse, which produces the seed with $2.44 \mu \mathrm{J}$ energy in Fig. 7 is used in the longbunch simulations.

The FEL calculations are performed within a $6 \sigma_{\mathrm{e}}$ time window, where $\sigma_{\mathrm{e}}$ is the rms duration of the electron bunch. The current and energy distributions along the electron bunch are assumed to be Gaussian. Calculations in the U2 FEL system are performed using a fresh bunch, so as not to obscure the self-seeding effects by additional energy spread after the U1 FEL system. Using a fresh bunch in the U2 FEL system is actually a very attractive option from many points of view. Use of the fresh bunch has 


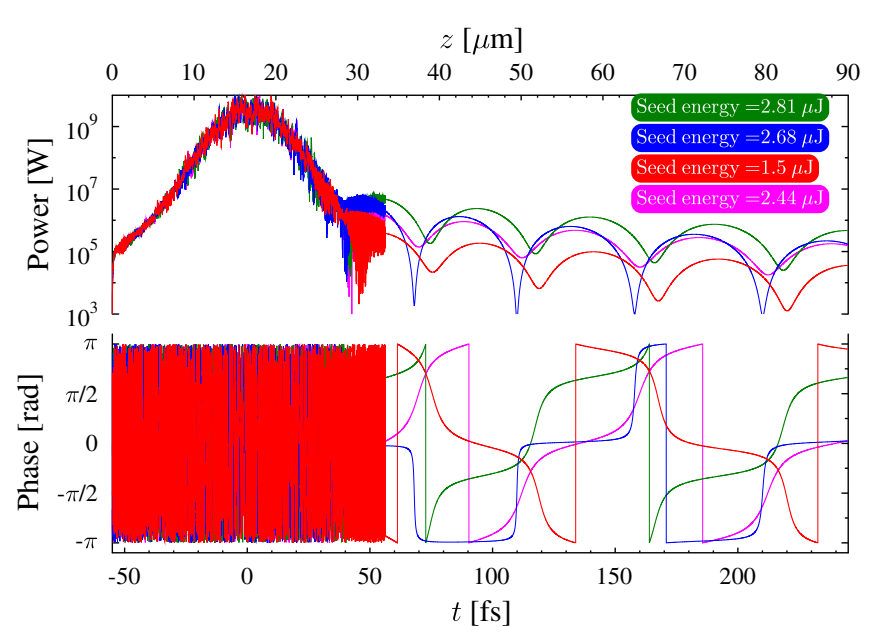

FIG. 7. Time dependencies of power and phase of the entire radiation interacting with the electron bunch at the beginning of the $\mathrm{U} 2$ undulator system. The entire radiation comprises both the SASE radiation [the first term of Eq. (5)], and the monochromatic seed generated by FBD of the SASE radiation from a crystal [the second term of Eq. (5)]. Examples of different SASE pulses in the FEL long-pulse operation mode are shown, demonstrating jitter in the seed power, and positions of the seed maxima. The crystal monochromator with $\mathcal{T}_{0}=0.75 \mathrm{fs}$ is used.

been discussed in the two-electron-bunch self-seeding scheme [19].

For simplicity it is also assumed that the emittance does not change along the bunch length. The idealized case we are considering here will allow us to make general qualitative conclusions. However, these conclusions may break down under more realistic conditions.

The orange lines in Figs. 8-11 show the electron current profile within the $6 \sigma_{\mathrm{e}}$ time window. The dashed cyan lines represent the SASE radiation output after the $\mathrm{U} 1$ undulator system within the same time window. The solid magenta lines represent the pure monochromatic seed-the intensity of the second term in Eq. (5), calculated for FBD with different $\mathcal{T}_{0}$ values. The dashed blue lines represent the entire radiation, the sum of the SASE and of the monochromatic seed, i.e., everything that enters the U2 undulator system. The phase of the seed is shown in the graphs in the $\beta$ rows of Figs. 10 and 11 .

The seeded power at the end of the U2 FEL system is very sensitive to the normalized undulator parameter $a_{w}=$ $(e / m c)\left(B_{w} / 2 k_{w}\right)$. Variation by $0.06 \%$ from the optimal value $a_{w}=2.4729$ results in a more than $50 \%$ drop of power in the long-bunch mode. Parameter $a_{w}$ is defined by the on-axis undulator field amplitude $B_{w}$, the undulator wave number $k_{w}=2 \pi / \lambda_{w}$, the electron rest mass $m$, and the elementary charge $e$.

\section{A. Short-bunch mode}

We first validate the method by using the short electron bunch. The wakefield effects from the undulator chamber
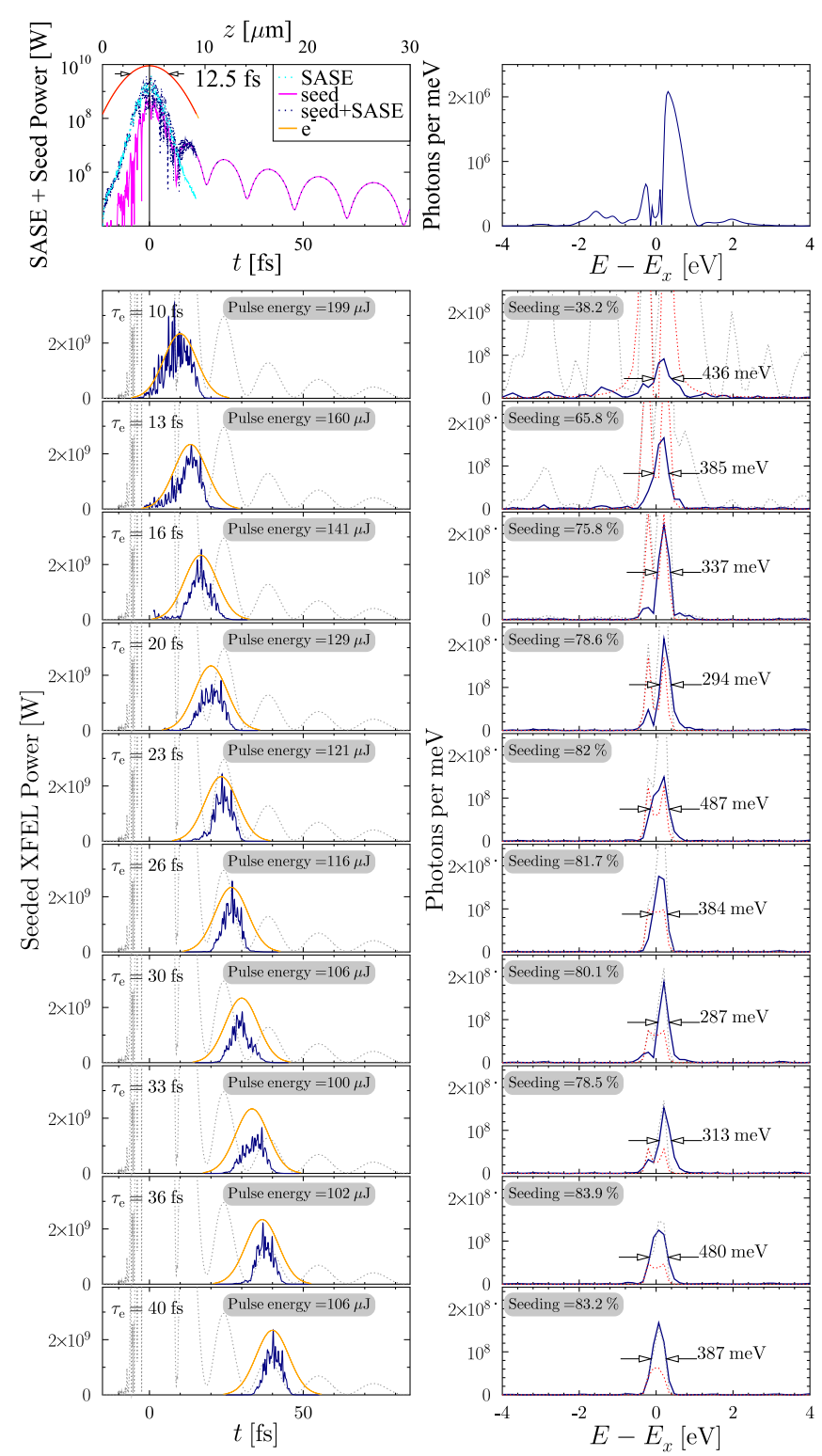

FIG. 8. Time (left) and spectral (right) dependencies of the XFEL radiation in the self-seeding short-bunch mode. Graphs in the top row present the dependencies at the entrance of the U2 undulator system. Seeding is performed by the FBD monochromator with the FBD time constant $\mathcal{T}_{0}=0.21$ fs. The top right spectrum of the seeding radiation is time integrated. The lower graphs show in solid dark blue lines the time and spectral dependencies at the end of the $\mathrm{U} 2$ system for different electron bunch delays $\tau_{e}$. Orange lines show the electron current profiles. The dashed gray lines in the left graphs are the time profile of the seed. The dashed gray lines in the right graphs represent the seed spectrum as it is seen by the electron bunch at appropriate time delays. Red dashed lines show the same, however, for an idealistic case of instantaneous excitation of the crystal with a white spectrum. The nominal photon energy $E_{x}=8.3 \mathrm{keV}$.

are ignored. The main XFEL parameters are presented in Table II.

Figure 8 shows an example of calculations with a monochromatic seed produced by filtering the SASE radiation 


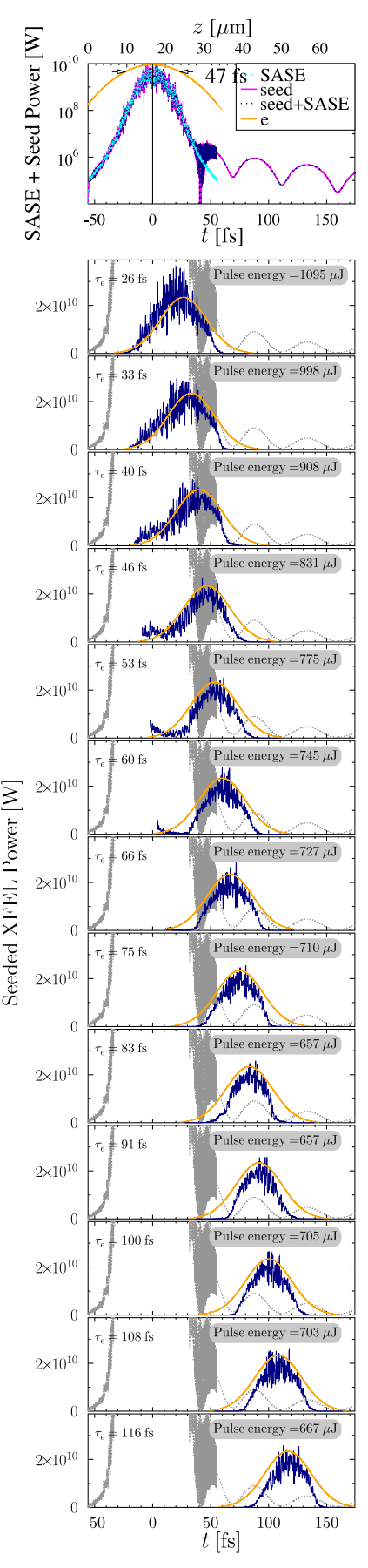

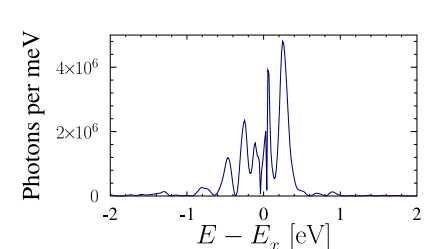
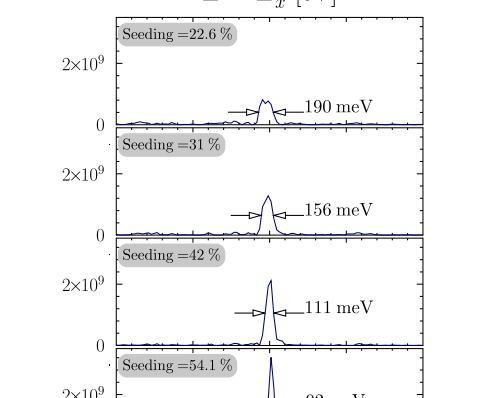

$$
\begin{aligned}
& \text { spectrum of the seed, and change with the delay } \tau_{e} \text {. We } \\
& \text { will present and discuss the results of the simulations in } \\
& \text { detail in Sec. IV. }
\end{aligned}
$$

\section{B. Long-bunch mode}

Having validated the simulations in the short-bunch mode, we apply the procedure to the long-bunch mode. The current and energy distributions along the bunch are also Gaussian. For simplicity, the wakefield effects due to the resistive wall impedance of the undulator chamber are not considered here as well.

In the short-bunch mode, the wakefield effects are negligible. However, in the long-bunch mode, they may induce a substantial energy chirp along the electron bunch, move electrons off the FEL interaction bandwidth, and thus degrade the seeding efficiency. There are several techniques that could be used to mitigate the wakefield effects: electron bunch prechirp [28], specially shaped vacuum chamber [29], linac optimization especially the rf phase, etc.

Figure 9 shows an example of calculations with a monochromatic seed produced by filtering the SASE radiation through the FBD monochromator with the FBD time constant $\mathcal{T}_{0}=0.75$ fs. Such a time constant could be realized in different ways, one of them presented in Table I: by using the 004 Bragg diffraction of the $9.1 \mathrm{keV}$ x rays from a $d=100 \mu \mathrm{m}$ thick diamond crystal in the symmetric Bragg-case geometry with an asymmetry angle $\eta=0$, a Bragg angle of $\theta=56.8^{\circ}$, and an extinction length of $\bar{\Lambda}_{H}^{(\mathrm{s})}=3.63 \mu \mathrm{m}$.

One of the striking features of the data presented in Fig. 9 is the substantial variation of the seeded radiation bandwidth with the electron bunch delay $\tau_{e}$. We will present and discuss the results of the simulations in detail in Sec. IV.

\section{RESULTS AND DISCUSSION}

The seeded XFEL radiation characteristics for different seeding conditions are presented graphically in the 
$z[\mu \mathrm{m}]$

$z[\mu \mathrm{m}]$
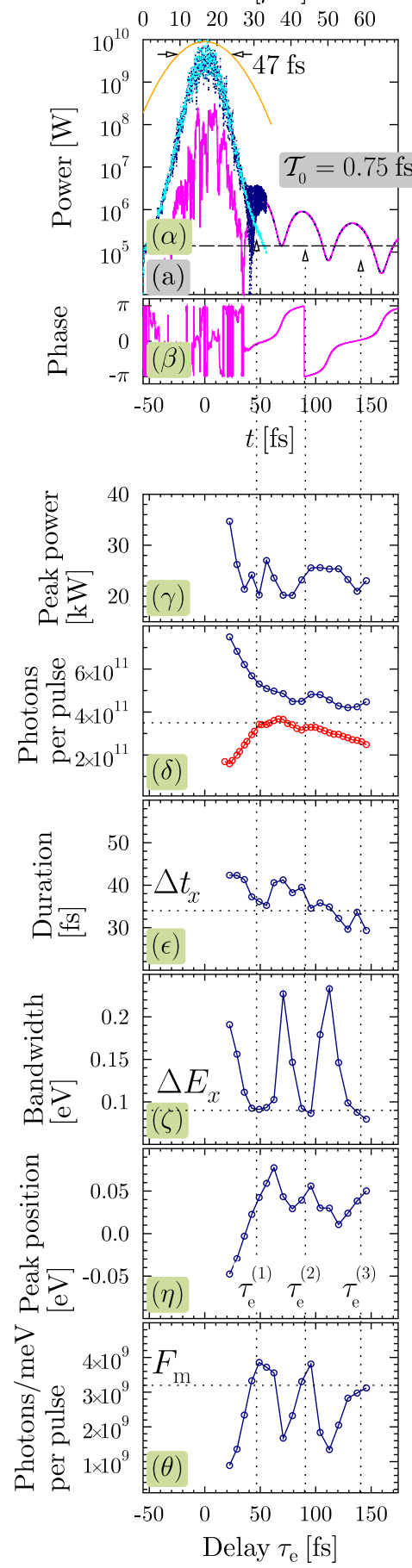
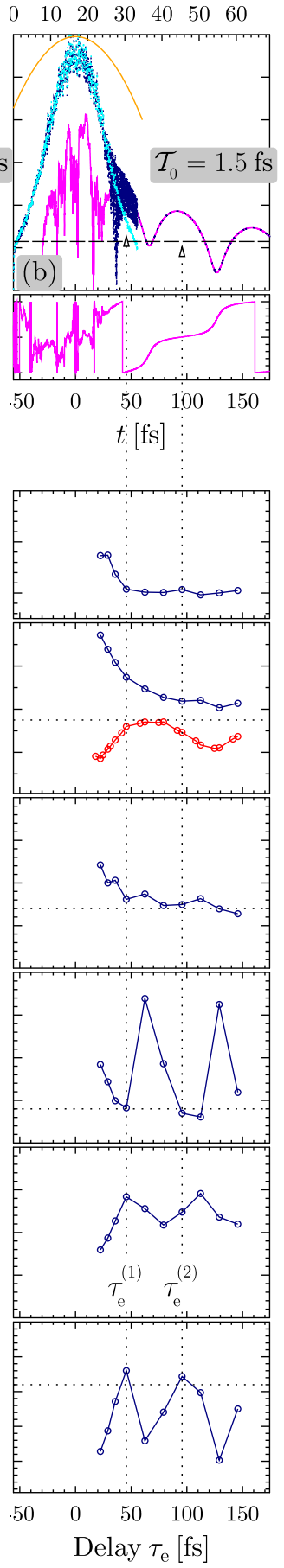

$z[\mu \mathrm{m}]$
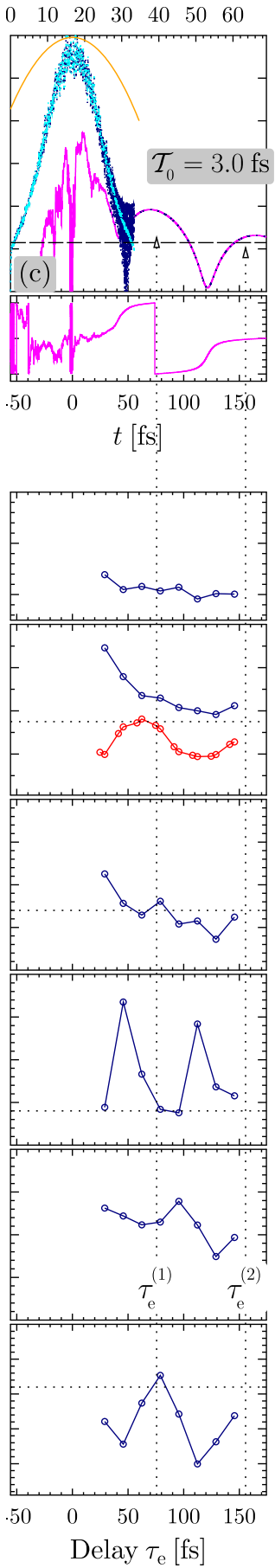

$z[\mu \mathrm{m}]$

$z[\mu \mathrm{m}]$
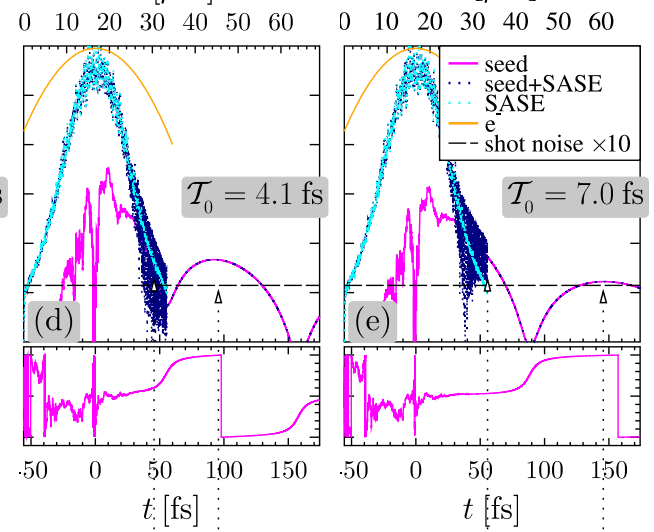

FIG. 10. Time dependencies of the monochromatic seed power (magenta lines in the $\alpha$-row graphs), seed plus SASE power (dashed blue line in same graphs), 10 times increased shot-noise level (dashed black line), and the time dependencies of the seed phase-graphs in the $\beta$ row. The seed is generated in the long-bunch mode by a SASE radiation from the U1 undulator system (cyan lines in the $\alpha$-row graphs) in forward Bragg diffraction (FBD) from crystals with FBD time constant $\mathcal{T}_{0}=0.75$ fs (a), $\mathcal{T}_{0}=1.5$ fs (b), $\mathcal{T}_{0}=3.0 \mathrm{fs}(\mathrm{c}), \mathcal{T}_{0}=4.1 \mathrm{fs}(\mathrm{d})$, and $\mathcal{T}_{0}=8 \mathrm{fs}(\mathrm{e})$. Graphs in other rows represent properties of the radiation at the end of the undulator system $\mathrm{U} 2$, as a function of the electron bunch delay $\tau_{e}$ in the magnetic chicane.

long-bunch mode in Fig. 10 and in the short-bunch mode in Fig. 11. The seeding conditions are varied by changing FBD time constant $\mathcal{T}_{0}$ and the electron bunch delay $\tau_{e}$. The results for different $\mathcal{T}_{0}$ are shown in the appropriate columns of Figs. 10 and 11. The XFEL radiation characteristics (the focus is on spectral properties) such as peak power, total number of photons, total number of seeded photons, x-ray pulse duration, spectral bandwidth, and 

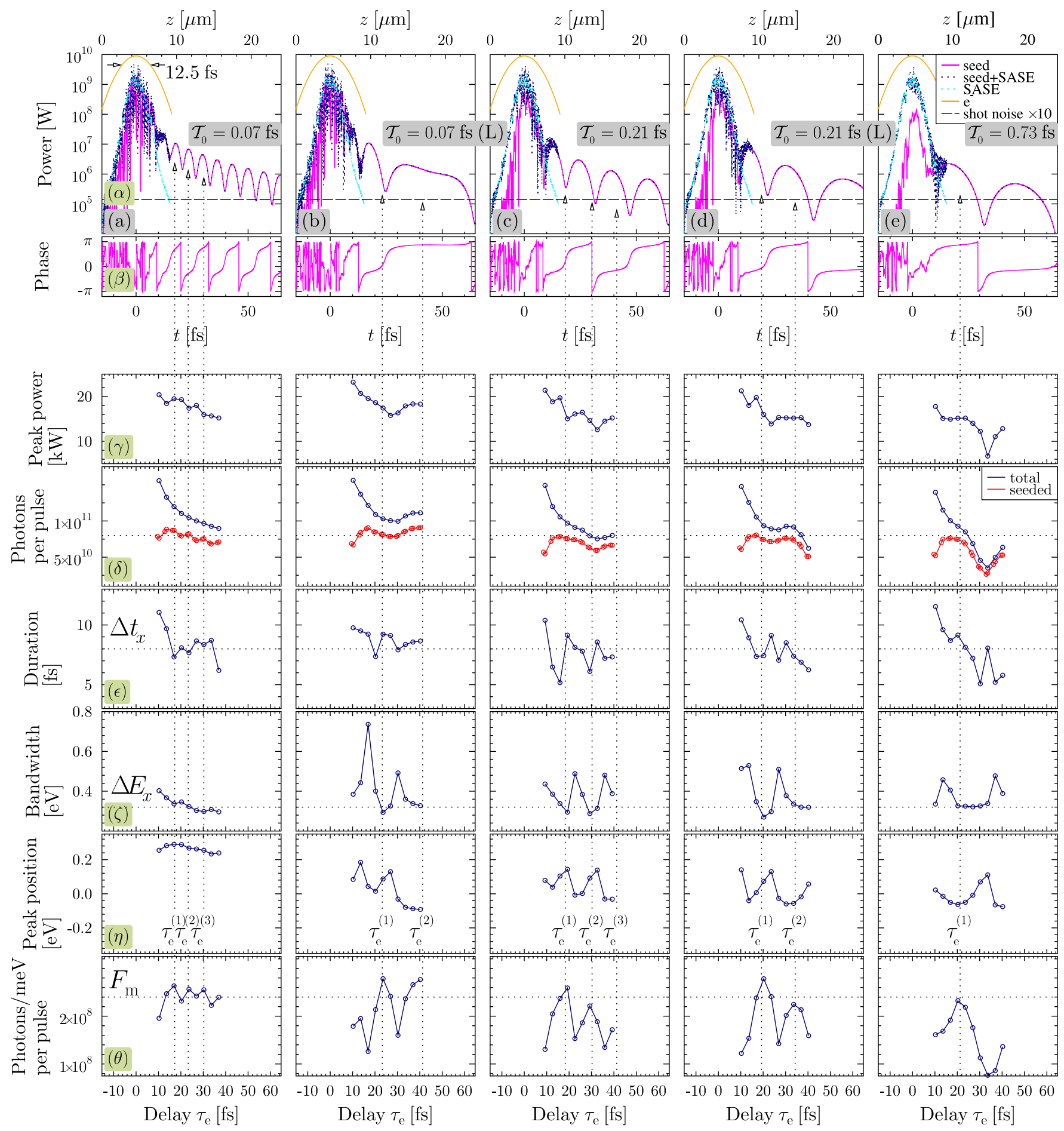

FIG. 11. Same as Fig. 10, but for the short-bunch mode calculations with (a) $\mathcal{T}_{0}=0.07 \mathrm{fs}$ (Bragg case), (b) $\mathcal{T}_{0}=0.07 \mathrm{fs}$ (Laue case), (c) $\mathcal{T}_{0}=0.21 \mathrm{fs}$ (Bragg case), (d) $\mathcal{T}_{0}=0.21 \mathrm{fs}$ (Laue case), and (e) $\mathcal{T}_{0}=0.73 \mathrm{fs}$ (Bragg case).

spectral line center position are presented as a function of the electron bunch delay $\tau_{e}$ by the graphs in respective rows of Figs. 10 and 11. $\mathcal{T}_{0}$-independent characteristics of the XFEL radiation are presented in Table III.

The main observations, which can be derived from the results presented in Figs. 10 and 11, are summarized below.

\section{A. Peak power}

The peak power of the radiation pulse at the end of the XFEL is stable, and independent of $\mathcal{T}_{0}$ and of the electron bunch delay $\tau_{e}$. See graphs in the $\gamma$ rows. It has approximately the same magnitude, both in the short-bunch and in the long-bunch modes. Still, it is slightly larger in the 
TABLE III. $\mathcal{T}_{0}$-independent XFEL radiation parameters in self-seeding mode with FBD monochromators. By "seeded radiation" we define the portion of the total radiation enclosed within the narrow bandwidth, as a result of self-seeding process.

\begin{tabular}{|c|c|c|c|}
\hline Parameter and symbol & Long-bunch mode & Short-bunch mode & Unit \\
\hline Photon energy $E_{\mathrm{x}}$ & 9.10 & 8.30 & $\mathrm{keV}$ \\
\hline Wavelength & 1.36 & 1.50 & $\AA$ \\
\hline \multicolumn{4}{|l|}{ SASE radiation after $\mathrm{U} 1$} \\
\hline SASE pulse energy & 83 & 9 & $\mu \mathrm{J}$ \\
\hline SASE peak power & 3 & 1.2 & GW \\
\hline \multicolumn{4}{|l|}{ Seeded radiation after U2 } \\
\hline Pulse peak power & 20 & 15 & \multirow[t]{2}{*}{ GW } \\
\hline Photons per pulse & $4 \times 10^{11}$ & $8 \times 10^{10}$ & \\
\hline Pulse energy & 680 & 100 & $\mu \mathrm{J}$ \\
\hline Pulse duration $\Delta t_{\mathrm{x}}$ & 34 (FWHM) & 8 (FWHM) & fs \\
\hline Pulse duration $\sigma_{\mathrm{x}}$ & 15 (rms) & $3.5(\mathrm{rms})$ & fs \\
\hline Minimum bandwidth $\Delta E_{\mathrm{x}}$ & 90 (FWHM) & 320 (FWHM) & $\mathrm{meV}$ \\
\hline Minimum bandwidth $\sigma_{\mathrm{E}_{\mathrm{x}}}$ & $38(\mathrm{rms})$ & $136(\mathrm{rms})$ & $\mathrm{meV}$ \\
\hline Maximum spectral flux $F_{\mathrm{m}}$ & $\simeq 3 \times 10^{9}$ & $\simeq 2 \times 10^{8}$ & $\mathrm{ph} / \mathrm{meV}$ \\
\hline$F_{\mathrm{m}}$ per pC, $\bar{F}_{\mathrm{m}}$ & $\simeq 2 \times 10^{7}$ & $\simeq 6 \times 10^{6}$ & $\mathrm{ph} / \mathrm{meV} / \mathrm{pC}$ \\
\hline
\end{tabular}

long-bunch mode. This is consistent with the FEL theory, see Eq. (91) of [16], predicting the saturation power level $P_{\text {sat }} \propto \rho P_{\text {beam }}$ to be a fraction of the total electron beam power $P_{\text {beam }}=E_{\mathrm{e}} I_{\mathrm{e}} / e$, with the fraction given by the dimensionless Pierce parameter $\rho$ [13], which is typically $\simeq 10^{-3}$. The peak current $I_{\mathrm{e}}$ and $\rho$ values are the same in both cases, however, the electron energy $E_{\mathrm{e}}$ is chosen larger in the long-bunch mode, see Table II.

\section{B. Number of photons per pulse}

The total number of photons per pulse changes with the delay $\tau_{e}$. It is the largest at smaller delays, where the SASE contribution is the largest, see graphs in the $\delta$ rows of Figs. 10 and 11. On average, the total number of photons is 4 times larger in the long-bunch mode, which corresponds to a 4 times larger pulse duration and 4 times larger charge in the long-bunch compared to the short-bunch mode.

We are interested primarily in the total number of "seeded photons," those condensed in a narrow bandwidth, as a result of the self-seeding process. The bandwidth may change with $\mathcal{T}_{0}$, as seen in the right columns of Figs. 8 and 9. The total number of seeded photons within a band defined as the four smallest bandwidths is shown by red circles in $\delta$-row graphs of Figs. 10 and 11 . The total number of seeded photons is largest for those electron bunch delays $\tau_{e}$ at which the electron bunch is seeded with the radiation (magenta lines in the $\alpha$-row graphs of Figs. 10 and 11) whose power is much larger than the shot-noise power $P_{\mathrm{n}}$ level. Dashed black lines in the $\alpha$-row graphs of Figs. 10 and 11 show the $10 \times P_{\mathrm{n}}$ level. The effective shot-noise power is estimated to be $P_{\mathrm{n}} \simeq$ $\rho^{2} E_{\mathrm{e}} E_{\mathrm{x}} / \hbar \sqrt{2 \pi} \simeq 14 \mathrm{~kW}[14,16]$.

\section{Pulse duration}

The duration of the x-ray pulse $\Delta t_{\mathrm{x}}(\mathrm{FWHM})$ is shorter than the duration of the electron bunch; see Figs. 8 and 9, and the graphs in the $\epsilon$ rows of Figs. 10 and 11. This fact reflects the nonlinear nature of lasing physics, in which the high-current portion of the electron bunch produces the most significant effect.

\section{Spectral bandwidth}

The spectral bandwidth of the radiation achieves its smallest values $\Delta E_{\mathrm{x}}$ (FWHM) at specific electron bunch delays $\tau_{e}^{(n)}$, with respect to the monochromatic seed, indicated by vertical dashed lines with arrows; see the $\zeta$-row graphs in Figs. 10 and 11. The smallest achievable bandwidth $\Delta E_{\mathrm{x}}$ is practically independent of $\mathcal{T}_{0}$. However, it takes different values in the long-bunch and in the shortbunch modes.

The product $\sigma_{\mathrm{x}} \sigma_{\mathrm{E}_{\mathrm{x}}} \approx 5 \times 10^{-16} \mathrm{eV} \cdot \mathrm{s}$ of the $\mathrm{rms}$ $\mathrm{x}$-ray pulse duration, and the rms minimal $\mathrm{x}$-ray pulse bandwidth is very close to Planck's constant $\hbar=6.6 \times 10^{-16} \mathrm{eV} \cdot \mathrm{s}$. This suggests that self-seeding results in the almost Fourier-transform limited, i.e., in the fully longitudinally coherent, FEL radiation. This also very clearly suggests that the ultimate spectral properties of the self-seeding XFEL are determined first of all by the length $\sigma_{\mathrm{e}}$ of the electron bunch rather than by the properties of the radiation seed. The seed helps to achieve the best performance; however, it does not determine the final result.

The bandwidth may increase by a factor of 3 from its smallest value if the electron bunch delay is chosen somewhere between the specific delays $\tau_{e}^{(n)}$; see Fig. $10(\eta)$. 
The larger $\mathcal{T}_{0}$ is, the broader are the $\tau_{e}$ intervals where the smallest bandwidth values are attained. Because of the jitter in the SASE radiation, resulting in a jitter of the positions and of the seed power peaks and phase (see Fig. 7), it may be more advantageous to choose FBD with longer $\mathcal{T}_{0}$ values, to achieve spectrally stable selfseeding with narrowest bandwidth and highest spectral flux, independent of SASE jitter. At the same time adequate seed power should be ensured as well. FBD with too long a $\mathcal{T}_{0}$ may produce insufficient seed power. The optimal value is $\mathcal{T}_{0} \simeq \sigma_{\mathrm{e}} / \pi$, as was discussed in Sec. II B. FBD with a very short $\mathcal{T}_{0} \ll \sigma_{\mathrm{e}} / \pi$ may result in double-color seeding.

\section{E. Double-color seeding}

Double-color seeding in the long-bunch mode is shown in Fig. 12, with an FBD monochromator having $\mathcal{T}_{0}=0.07$ fs. We note that the double-color seeding with equal intensities of both colors, as shown in Fig. 12, cannot be achieved in each XFEL shot. Our simulations show that the relative intensity variations of the two colors take place from shot to shot. The success for equal-intensity doublecolor seeding depends on the spectral composition of the SASE spectrum. Only if both sharp peaks of the FBD spectrum, as in Fig. 6(b), are "populated," equal-intensity double-color seeding does take place. The total power of the seeded radiation is the same as in single-color seeding; however, it is shared between the two spectral components.

The nonlinear chirp may heavily impact the self-seeding output spectral shape even in the case of a short bunch. As a result, the double-color spectrum with separation of the features on a sub-eV-scale may to be washed out. However, understanding these details needs more comprehensive studies.

\section{F. Spectral line center position}

The center of the self-seeding XFEL output spectrum varies with the electron bunch delay, as shown in the $\eta$-row graphs of Figs. 10 and 11. The variation is not pronounced; however, it correlates with the time structure of the monochromatic seed.

A considerable shift of $\simeq+0.2 \mathrm{eV}$ of the peak position in the $\mathcal{T}_{0}=0.07$-fs Bragg case in the short-bunch mode is due to the fact that this is actually the double-color seeding case. There are actually two sharp peaks in this case FBD seeding spectrum, as in Fig. 6. However, for the particular SASE spectrum used in these simulations, only one of the two peaks is populated.

\section{G. Spectral flux}

In each mode, the maximum spectral flux $F_{\mathrm{m}}$ (measured in photons/pulse/meV) is independent of $\mathcal{T}_{0}$; see the $\eta$-row graphs in Figs. 10 and 11. In the long-bunch mode $F_{\mathrm{m}} \simeq 3.2 \times 10^{9} \mathrm{ph} / \mathrm{meV} /$ pulse, while in the short-bunch
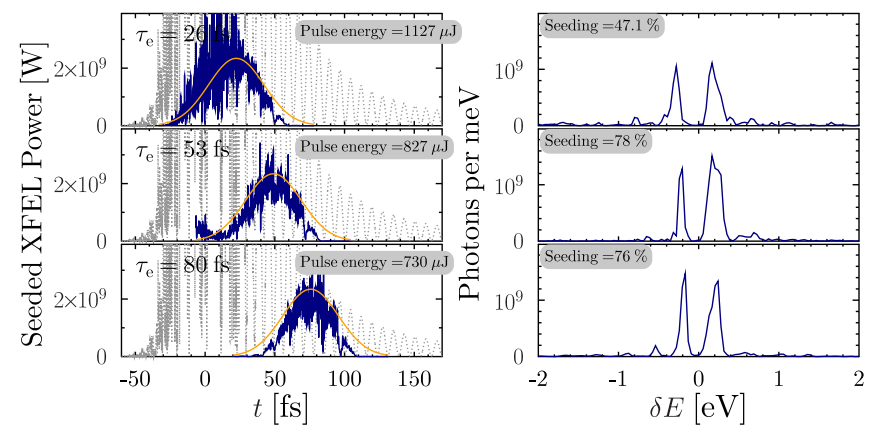

FIG. 12. Time (left) and spectral (right) dependencies of the XFEL radiation in the self-seeding long-bunch mode with the FBD monochromator having $\mathcal{T}_{0}=0.07$ fs. The graphs show in solid dark blue lines the time and spectral dependencies at the end of the U2 system for different electron bunch delays $\tau_{e}$. Orange lines show the electron current profiles. The dashed gray lines in the left graphs are the time profiles of the seed. The nominal photon energy $E_{x}=9.1 \mathrm{keV}$.

mode it is an order of magnitude smaller $-F_{\mathrm{m}} \simeq$ $2.2 \times 10^{8} \mathrm{ph} / \mathrm{meV} /$ pulse. The spectral flux values, normalized to the total bunch charge, are $\bar{F}_{\mathrm{m}} \simeq 2.1 \times$ $10^{7} \mathrm{ph} / \mathrm{meV} / \mathrm{pC} / \mathrm{pulse}$ in the long-bunch mode and $\bar{F}_{\mathrm{m}} \simeq 0.55 \times 10^{7} \mathrm{ph} / \mathrm{meV} / \mathrm{pC} /$ pulse in the short-bunch mode, respectively, and differ by a factor of 4 . For comparison, an XFEL in the oscillator configuration produces $\bar{F}_{\mathrm{m}} \simeq 2 \times 10^{7} \mathrm{ph} / \mathrm{meV} / \mathrm{pC} /$ pulse [30], the number close to the high-gain XFEL in the long-bunch mode considered here.

The maximum spectral flux is attained at the same specific electron bunch delays $\tau_{e}^{(n)}$ at which the smallest spectral bandwidth is achieved. These delays correlate but do not always coincide with the seed power peaks in the $\alpha$-row graphs. The delays $\tau_{e}$ are systematically shifted to later times from the peaks' positions. This is clearly seen especially in the short-pulse mode examples in Fig. 11.

The efficient seeding can be performed by using the first or second trailing peaks of the FBD radiation, as discussed in this paper and in the literature $[1,21]$. However, it also can be performed efficiently by using the zeroth maximum of the FBD radiation, as the case of $\mathcal{T}_{0}=7 \mathrm{fs}$ in the longbunch mode in Fig. $10(\eta)$ demonstrates, with the delay set at $\tau_{e} \simeq 50 \mathrm{fs}$.

Figure $11(\eta)$, for $\mathcal{T}_{0}=0.07 \mathrm{fs}$, shows an exceptional case of seeding, resulting in high spectral flux and smallest bandwidth practically independent of the delay $\tau_{e}$. This does not fit into the general picture discussed until now. Indeed, this is achieved under exceptional conditions, when the period of the FBD power oscillations is much shorter than the electron bunch duration, i.e., under the conditions where double-color seeding is expected. As was already mentioned in Sec. IVF, there are actually two sharp peaks in the FBD seeding spectrum in this case, as in Fig. 6. However, the particular SASE spectrum used in 
the simulations has spectral components which "populate" only one of the of the two FBD peaks.

The case $\mathcal{T}_{0}=0.07 \mathrm{fs}$ proves again that a strong seed will produce a stable $\tau_{e}$-independent output with the spectral flux and spectral width defined eventually by the XFEL parameters, rather than by the seed properties.

In Sec. II we have stated that for time delays $t \ll \mathcal{T}_{d}$, the Bragg-case and Laue-case FBDs are equivalent and are defined by a single parameter-the FBD time constant $\mathcal{T}_{0}$; see Eq. (9). At longer times, however, Bragg-case and Laue-case FBDs are not equivalent, and have to be described by different equations (6) and (7), respectively, with the time constant $\mathcal{T}_{d}$ taken into account. Examples of the $\mathcal{T}_{0}=0.07 \mathrm{fs}$ Bragg case and the $\mathcal{T}_{0}=0.07 \mathrm{fs}$ Laue case, in the short-bunch mode, with $\mathcal{T}_{d}=94 \mathrm{fs}$ (see Table I), demonstrate substantial differences between the two cases already for time delays $t>30 \mathrm{fs}$. Bragg-case FBD results in double-color seeding. On the contrary, Laue-case FBD ensures single-color seeding with very good, if not the best, results for spectral density and spectral width, due to a strong signal and large duration of the seed at $t=40 \mathrm{fs}$.

\section{APPLICATIONS}

Self-seeding XFELs can generate fully coherent x-ray pulses with a very high spectral flux, much higher than the state-of-the-art storage ring-based synchrotron radiation sources. Their supreme spectral properties will expand the science reach of the XFELs and stimulate the utilization of advanced high-resolution spectroscopic techniques.

A self-seeding XFEL, in the long-bunch mode considered in this paper, can generate in each pulse $\simeq 4 \times 10^{11}$ photons-see $\delta$-row graphs of Fig. 10 - concentrated within a $90-\mathrm{meV}$ (FWHM) bandwidth, or $\simeq 3 \times 10^{9}$ photons $/ \mathrm{meV}$ $\operatorname{see} \theta$-row graphs of Fig. 10 . With the $120-\mathrm{Hz}$ repetition rate of the LCLS machine, the average number of photons that can be generated in a $90-\mathrm{meV}$ (FWHM) bandwidth is $\simeq 4.8 \times 10^{13}$ photons $/ \mathrm{s}$, corresponding to a spectral flux of $\simeq 3.5 \times 10^{11}$ photons $/ \mathrm{s} / \mathrm{meV}$. This spectral flux number is an order of magnitude larger than an average spectral flux available at the 3rd generation storage ring-based machines such as ESRF, APS, or SPring-8. The future high-repetitionrate XFELs, such as the European XFEL [31], with an average $30-\mathrm{kHz}$ pulse rate, will be able to generate a spectral flux of $\simeq 8.7 \times 10^{13}$ photons $/ \mathrm{s} / \mathrm{meV}$, i.e., approximately 3 orders of magnitude more than the average spectral flux available presently at the synchrotron radiation facilities.

High-resolution inelastic $x$-ray scattering (IXS) and resonant IXS (RIXS) spectroscopies, widely used for studies of collective excitation in condensed matter [32-34], will tremendously benefit from $\mathrm{x}$-ray sources with largely enhanced average and peak spectral flux. In particular, applications of IXS and RIXS spectrographs working with $9-\mathrm{keV}$ photons $[35,36]$ will permit studies with spectral resolution better than $0.1 \mathrm{meV}$. X-ray sources with largely enhanced average spectral flux would be also attractive for other high-spectral-resolution tools, such as nuclear resonance (Mössbauer) spectroscopies [37,38], as was discussed, in particular, in the first paper on hard x-ray self-seeding [18].

\section{CONCLUSIONS}

We have performed theoretical studies for the best conditions under which spectrally stable self-seeding of the hard x-ray FEL can be achieved with smallest shot-to-shot variations of the output radiation, with narrowest bandwidth, and largest spectral flux. Characteristic time $\mathcal{T}_{0}$ of forward Bragg diffraction (FBD) in the x-ray monochromator crystal determines the power, spectral, and time characteristics of the FBD monochromatic seed. For a given electron bunch duration $\sigma_{\mathrm{e}}$ the spectral flux of the self-seeding XFEL can be maximized, the spectral bandwidth can be respectively minimized, by choosing $\mathcal{T}_{0} \sim \sigma_{\mathrm{e}} / \pi$, and by optimizing the electron bunch delay $\tau_{e}$. The choice of $\mathcal{T}_{0}$ and $\tau_{e}$ is not unique. In all cases, the maximum value of the spectral flux and the minimum bandwidth are primarily determined by the electron bunch length $\sigma_{\mathrm{e}}$. The longer the electron bunch, the higher spectral flux can be achieved. It is important, however, that the power of the seed is about 2 orders of magnitude larger than the shot-noise power, which calls for using FBD monochromators with smaller $\mathcal{T}_{0}$ values. However, if $\mathcal{T}_{0} \ll \sigma_{\mathrm{e}} / \pi$ is very short, this may result in two-color seeding.

\section{ACKNOWLEDGMENTS}

We gratefully acknowledge useful discussions with ZhiRong Huang, Gregory Penn, L.H. Yu, and Sven Reiche. ZhiRong Huang and Juhao Wu are acknowledged for providing LCLS FEL parameters. Work at Brookhaven National Laboratory was supported by the U.S. Department of Energy, Office of Science, Office of Basic Energy Sciences, under Contract No. DE-AC02-98CH1886. Work at Argonne National Laboratory was supported by the U.S. Department of Energy, Office of Science, under Contract No. DE-AC02-06CH11357.

[1] J. Amann, W. Berg, V. Blank, F.-J. Decker, Y. Ding, P. Emma, Y. Feng, J. Frisch, D. Fritz, J. Hastings et al., Nat. Photonics 6, 693 (2012).

[2] E. Allaria, R. Appio, L. Badano, W. Barletta, S. Bassanese, S. Biedron, A. Borga, E. Busetto, D. Castronovo, P. Cinquegrana et al., Nat. Photonics 6, 699 (2012).

[3] A. T. Lin and J. M. Dawson, Phys. Rev. Lett. 42, 1670 (1979).

[4] P. Sprangle, C.-M. Tang, and W. M. Manheimer, Phys. Rev. Lett. 43, 1932 (1979).

[5] N. Kroll, P. Morton, and M. Rosenbluth, IEEE J. Quantum Electron. 17, 1436 (1981). 
[6] T. J. Orzechowski, B. R. Anderson, J.C. Clark, W. M. Fawley, A. C. Paul, D. Prosnitz, E. T. Scharlemann, S. M. Yarema, D. B. Hopkins, A. M. Sessler et al., Phys. Rev. Lett. 57, 2172 (1986).

[7] W. M. Fawley, Z. Huang, K.-J. Kim, and N. A. Vinokurov, Nucl. Instrum. Methods Phys. Res., Sect. A 483, 537 (2002).

[8] X. J. Wang, H.P. Freund, D. Harder, W. H. Miner, J. B. Murphy, H. Qian, Y. Shen, and X. Yang, Phys. Rev. Lett. 103, 154801 (2009).

[9] G. Geloni, V. Kocharyan, and E. Saldin, arXiv:1007.2743.

[10] W. Fawley, J. Frisch, Z. Huang, Y. Jiao, H.-D. Nuhn, C. Pellegrini, S. Reiche, and J. Wu, SLAC National Accelerator Laboratory Technical Report No. SLACPUB-14616, 2011.

[11] Y. Jiao, J. Wu, Y. Cai, A.W. Chao, W. M. Fawley, J. Frisch, Z. Huang, H.-D. Nuhn, C. Pellegrini, and S. Reiche, Phys. Rev. ST Accel. Beams 15, 050704 (2012).

[12] Y.S. Derbenev, A. M. Kondratenko, and E. L. Saldin, Nucl. Instrum. Methods Phys. Res. 193, 415 (1982).

[13] R. Bonifacio, C. Pellegrini, and L. Narducci, Opt. Commun. 50, 373 (1984).

[14] K.-J. Kim, Phys. Rev. Lett. 57, 1871 (1986).

[15] E. L. Saldin, E. A. Schneidmiller, and M. V. Yurkov, The Physics of Free Electron Lasers, Advanced Texts in Physics (Springer, Berlin, 2000).

[16] Z. Huang and K.-J. Kim, Phys. Rev. ST Accel. Beams 10, 034801 (2007).

[17] J. Feldhaus, E. Saldin, J. Schneider, E. Schneidmiller, and M. Yurkov, Opt. Commun. 140, 341 (1997).

[18] E. L. Saldin, E. A. Schneidmiller, Yu. V. Shvyd'ko, and M. V. Yurkov, Nucl. Instrum. Methods Phys. Res., Sect. A 475, 357 (2001).

[19] Y. Ding, Z. Huang, and R. D. Ruth, Phys. Rev. ST Accel. Beams 13, 060703 (2010).

[20] G. Geloni, V. Kocharyan, and E. Saldin, arXiv:1003.2548.

[21] G. Geloni, V. Kocharyan, and E. Saldin, J. Mod. Opt. 58, 1391 (2011).

[22] Yu. Shvyd'ko and R. Lindberg, Phys. Rev. ST Accel. Beams 15, 100702 (2012).

[23] S. Reiche, Nucl. Instrum. Methods Phys. Res., Sect. A 429, 243 (1999).
[24] R. R. Lindberg and Yu. V. Shvyd'ko, Phys. Rev. ST Accel. Beams 15, 050706 (2012).

[25] Yu. Shvyd'ko, X-Ray Optics-High-Energy-Resolution Applications, Optical Sciences (Springer, Berlin, 2004), Vol. 98.

[26] E. Saldin, E. Schneidmiller, and M. Yurkov, Opt. Commun. 148, 383 (1998).

[27] P. Emma, R. Akre, J. Arthur, R. Bionta, C. Bostedt, J. Bozek, A. Brachmann, P. Bucksbaum, R. Coffee, F.-J. Decker et al., Nat. Photonics 4, 641 (2010).

[28] M. Cornacchia, S. Di Mitri, G. Penco, and A. A. Zholents, Phys. Rev. ST Accel. Beams 9, 120701 (2006).

[29] K. L.F. Bane and G. Stupakov, Nucl. Instrum. Methods Phys. Res., Sect. A 690, 106 (2012).

[30] R. R. Lindberg, K.-J. Kim, Yu. Shvyd'ko, and W. M. Fawley, Phys. Rev. ST Accel. Beams 14, 010701 (2011).

[31] M. Altarelli, R. Brinkmann, M. Chergui, W. Decking, B. Dobson, S. Dusterer, G. Grübel, W. Graeff, H. Graafsma, J. Hajdu et al., XFEL: The European X-Ray Free-Electron Laser : Technical design report (DESY, Hamburg, 2006).

[32] E. Burkel, Rep. Prog. Phys. 63, 171 (2000).

[33] M. Krisch and F. Sette, Light Scattering in Solids IX, Topics in Applied Physics (Springer, Berlin, 2007), Vol. 108, pp. 317-370.

[34] L. J. P. Ament, M. van Veenendaal, T. P. Devereaux, J. P. Hill, and J. van den Brink, Rev. Mod. Phys. 83, 705 (2011).

[35] Yu. Shvyd'ko, Proc. SPIE Int. Soc. Opt. Eng. 8502, 85020J (2012).

[36] Yu. Shvyd'ko, S. Stoupin, K. Mundboth, and J. Kim, Phys. Rev. A 87, 043835 (2013).

[37] Special issue on Nuclear Resonant Scattering of Synchrotron Radiation, edited by E. Gerdau and H. de Waard [Hyperfine Interact., 123-125, 0 (2000)].

[38] R. Röhlsberger, Nuclear Condensed Matter Physics with Synchrotron Radiation. Basic Principles, Methodology and Applications, Springer Tracts in Modern Physics (Springer Verlag, Berlin, 2004), Vol. 208. 